\title{
PaL Diagrams: A Linear Diagram-Based Visual Language
}

\author{
Peter Chapman $^{1}$, Gem Stapleton ${ }^{1}$, Peter Rodgers ${ }^{2}$ \\ ${ }^{1}$ University of Brighton, ${ }^{2}$ University of Kent \\ E-mail: $\{$ p.b.chapman, g.e.stapleton\}@brighton.ac.uk, p.j.rodgers@kent.ac.uk
}

\begin{abstract}
Linear diagrams have recently been shown to be more effective than Euler diagrams when used for set-based reasoning. However, unlike the growing corpus of knowledge about formal aspects of Euler and Venn diagrams, there has been no formalisation of linear diagrams. To fill this knowledge gap, we present and formalise Point and Line ( $P a L)$ diagrams, an extension of simple linear diagrams containing points, thus providing a formal foundation for an effective visual language. We prove that PaL diagrams are exactly as expressive as monadic first-order logic with equality, gaining, as a corollary, an equivalence with the Euler diagram extension called spider diagrams. The method of proof provides translations between PaL diagrams and sentences of monadic first-order logic.
\end{abstract}

\section{Introduction}

Linear diagrams have a long history, with the first recorded use of them owing to Leibniz in 1686 [1, 4]. Much like Venn and Euler diagrams, they express information about sets in a visual way. Whilst Venn and Euler diagrams have been put on a formal footing (see [10]), linear diagrams have largely been overlooked, which we begin to address in this paper. In a linear diagram, parallel labelled line segments represent sets. The vertical overlap of lines represents the intersection of the corresponding sets. For example, consider the diagrams in Fig. 1. The three diagrams shown express the same information, namely that $A \cap B=\emptyset$, and $C \subseteq A: d_{1}$ is a Venn diagram, using shading to represent the emptiness of certain set intersections; $d_{2}$ is an Euler diagram, which uses disjointness of curves to represent emptiness of sets; and $d_{3}$ is a linear diagram, where the absence of any vertical overlap between the lines labelled $A$ and $B$ represents the emptiness of the corresponding set intersection.

As notations built upon Euler diagrams (hereafter Eulerbased diagrams) have been widely used and formalised, the expressiveness of these notations has been well stud- ied. Venn-II and Euler diagrams exactly as expressive as monadic first-order logic (MFOL) [12, 14]. Although it has not been formally established, the expressiveness of the Euler/Venn system is thought to be somewhere between MFOL and monadic first-order logic with equality $(\mathrm{MFOL}[=])$ [7]. Spider diagrams extend Euler diagrams with points, and are known to be exactly as expressive as MFOL $[=][16]$. Of this family of logics, generalised constraint diagrams are at least as expressive as dyadic firstorder logic making them the most expressive [13].

Recent research provided empirical evidence that linear diagrams can be more effective for visualisation than Euler-based diagrams. In the restricted setting of representing syllogisms, [11] showed that linear diagrams performed as well as Euler diagrams. In [3], where the context was general set-based reasoning, participants using linear diagrams outperformed those using Euler diagrams in terms of both task completion times and error rates. In order to exploit this interesting result, we propose an extension to linear diagrams, called $\mathrm{PaL}$ diagrams, by adding points allowing the representation of both sets and elements. We have two goals: $(a)$ to provide a formal foundation for $\mathrm{PaL}$ diagrams in order that we may $(b)$ determine contexts where these new diagrams maintain their advantage over Eulerbased notations. It is the first of these goals which is one focus of this paper.

Adequate formal foundations are important for a number of reasons. Firstly, to compare the efficacy of two notations, it is key that the two notations are capable of expressing the same information. Any meaningful comparison between notations can only be performed on information expressible in all. Without formalisation, determining the expressiveness of a notation is not possible. In this paper, we show that $\mathrm{PaL}$ diagrams are exactly as expressive as $\operatorname{MFOL}[=]$, giving us the corollary that $\mathrm{PaL}$ diagrams are equivalent in expressive power to spider diagrams, and more expressive than Euler and Venn-II diagrams. Secondly, while static diagrams are useful, the ability to manipulate and reason with diagrams in a coherent manner is also desirable. The only way in which such reasoning rules can be determined and shown to be sound is through formalisation. The develop- 

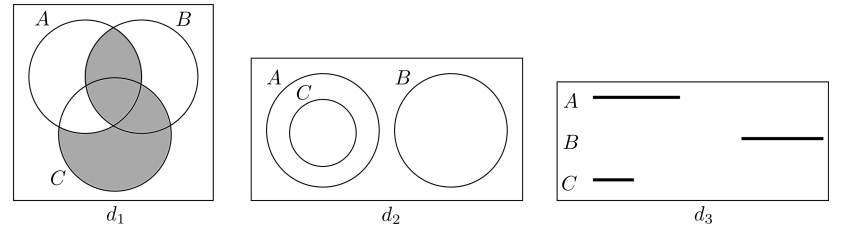

Figure 1. Venn, Euler and linear diagrams

ment of reasoning rules is outside the scope of this paper, but the work contained herein will allow such rules to be defined, and reasoned about, in a rigorous manner.

A number of notations have been derived from linear diagrams. For instance, the parallel bargrams of [18] and the double decker plots of [8] are both closely related to Leibniz's original version of linear diagrams, though neither are formal objects. To our knowledge, only one attempt has been made to formalise diagrams similar in flavour to linear diagrams, called line diagrams [6]. These diagrams contain non-parallel lines, and the intersection of these lines asserts the existence of an element in the corresponding sets. However, [9] showed the construction rules for these line diagrams were unsound. There is thus a gap for a formalization of linear diagrams, which is a key contribution of this paper.

The rest of the paper is organised as follows. In section 2 we give formal definitions of the syntax and semantics of PaL diagrams. Section 3 gives an overview of some MFOL[=] concepts necessary for establishing expressive equivalence of $\mathrm{PaL}$ diagrams and $\mathrm{MFOL}[=]$. The sections 4 and 5 contain demonstrations that every $\mathrm{PaL}$ diagram is equivalent to some sentence in $\operatorname{MFOL}[=]$, and that every sentence in MFOL $[=]$ can be equivalently expressed as a $\mathrm{PaL}$ diagram, respectively. We conclude and point to future directions in section 6 .

\section{PaL diagrams: Syntax and Semantics}

A PaL diagram consists of a set of parallel horizontal line segments (the actual orientation is somewhat irrelevant, all that is important is that the lines are parallel) with a collection of points arranged underneath the lines, as in Fig. 2. How the points and lines are arranged determines the meaning of the diagram. We proceed to present an abstract syntax for PaL diagrams.

In what follows, we take $\mathcal{L}$ to be a countably infinite set of letters, whose elements are called line labels, and $\mathcal{P}$ to be a countably infinite set of letters, whose elements are called point labels, disjoint from $\mathcal{L}$. In examples, we use capital roman letters $A, B, C, \ldots$ as elements of $\mathcal{L}$ and lower case roman letters $a, b, c, \ldots$ as elements of $\mathcal{P}$. When making general statements, we use $L_{i}$ and $p_{i}$ to denote line and

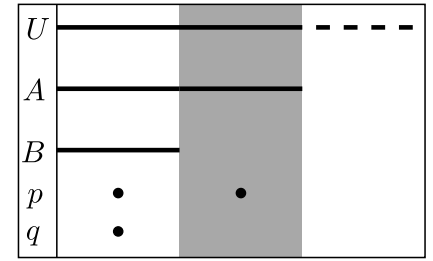

Figure 2. A PaL diagram

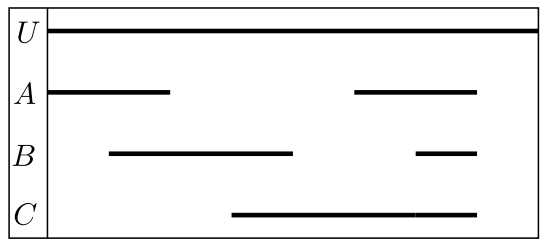

Figure 3. Venn-3

point labels respectively. We reserve the letter $U$ for a particular purpose, thus it is the case that $U \notin \mathcal{L} \cup \mathcal{P}$.

The main interaction between lines is the overlap which is where horizontal lines share the same vertical space. Formally:

Definition 1 An overlap is a word $L_{1} L_{2} \ldots L_{n}$ where each $L_{i}$ is a line label from $\mathcal{L}$, possibly adorned with a bar, $\bar{L}_{i}$. Given an overlap, $O$, we say that $L_{i}$ is barred if $\bar{L}_{i}$ appears in $O$, otherwise $L_{i}$ is unbarred. The set of all overlaps is denoted $\mathcal{O}$.

For example, suppose that $A, B$ and $C$ are line labels in $\mathcal{L}$. Then the following are overlaps: $A B C, \bar{A} B C$ and $A A \bar{B} C \bar{C}$. Note that last overlap is peculiar in that letters appear duplicated, barred and unbarred. We allow such overlaps to make reasoning about contradictions straightforward and intuitive, although that will be future work. In Fig. 2, there are three overlaps, reading left to right: $A B$ (where the lines labelled $A$ and $B$ overlap), $A \bar{B}$ (where the line $A$ does not overlap with $B$ ), and $\bar{A} \bar{B}$ (where neither $A$ nor $B$ appear). Notice that the top line, labelled $U$, does not appear in the overlaps. The line label $U$ is special: it represents the universal set and its presence indicates the extreme left and right coordinates of the line segments in the diagram. This limiting behaviour is important when there are overlaps whose letters are all barred; in Fig. 2, without the line for $U$, the PaL diagram could be taken to assert that all elements had to be in $A$, since the overlap $\bar{A} \bar{B}$ would be not be visible in the diagram.

Lines may consist of several segments. For example, in Fig. 3 we have a representation of Venn-3. This diagram would be impossible to draw without splitting at least one of the lines into segments. Here $A$ and $B$ consist of multiple 
segments, whereas $C$ consists of a single segment. The $U$ line can only consist of a single segment.

Overlaps can either be solid or dashed, and this is represented by - or $\cdots$... respectively. The interpretation of solid overlaps is that the set intersection represented is nonempty, whereas a dashed overlap could represent an empty set.

Points, which are visually drawn underneath overlaps as in Fig. 2, are formally defined in a similar way to overlaps, and to each overlap we associate the set of points occupying the same vertical space, called a clan of points. Formally:

Definition 2 A clan is a word $p_{1} p_{2} \ldots p_{n}$ where each $p_{i}$ is a letter drawn from $\mathcal{P}$, possibly adorned with a bar, $\bar{p}_{i}$. Given a clan cl, we say that $p_{i}$ is barred if $\bar{p}_{i}$ appears in $c l$, otherwise $p_{i}$ is unbarred.

For example, suppose that $a, b, c$ are letters from $\mathcal{P}$. Then the following are clans: $a \bar{b}, c \bar{c}$, and $a \bar{b} c$. Informally, points which are unbarred are said to lie under overlaps. In Fig. 2, there are three clans, reading left to right: $p q$ (where both $p$ and $q$ are under $A \bar{B}$ ), $p \bar{q}$ (where the point $p$ is under $A B$ but $q$ is not), and $\bar{p} \bar{q}$ (where neither $p$ nor $q$ are under $\bar{A} \bar{B}$ ).

PaL diagrams will comprise a list of overlaps, which may be solid or dashed, together with a set of clans. For (abstract) PaL diagrams to properly correspond to their concrete (drawn) realisations, it must be the case that each overlap and clan in a diagram is in some sense similar. We require that each overlap has the same underlying word. In order to formalise this, we introduce the rem function that removes bars from letters; in the definition below, $\lambda$ denotes the empty word and $\alpha_{i}$ denotes a single letter.

Definition 3 The remove function, rem : Overlap $\cup$ Clan $\rightarrow$ Overlap $\cup$ Clan,

is defined recursively by:

- $\operatorname{rem}(\lambda)=\lambda$,

- $\operatorname{rem}\left(\alpha_{i}\right)=\operatorname{rem}\left(\bar{\alpha}_{i}\right)=\alpha_{i}$,

- $\operatorname{rem}\left(\alpha_{i} \cdot w\right)=\operatorname{rem}\left(\alpha_{i}\right) \cdot \operatorname{rem}(w)$,

where $\cdot$ is the standard concatenation operator.

For example $\operatorname{rem}(\bar{A} B C)=A B C$ and $\operatorname{rem}(a \bar{b} c)=a b c$.

The last piece of syntax needed is shading. An overlap and clan sharing the same vertical space is either shaded or not shaded, indicated by $\square$ or $\square$, respectively. Shading is used to place an upper bound on the size of the set represented by the overlap.

Definition 4 A unitary PaL diagram, $d$, is a non-empty ordered list of 4-tuples (overlap $i$, type $_{i}$, clan $_{i}$, shading $_{i}$ ) such that:
- $\forall i, j \cdot \operatorname{rem}\left(\right.$ overlap $\left._{i}\right)=\operatorname{rem}\left(\right.$ overlap $\left._{j}\right)$,

- type $_{i} \in\{\cdots,-\}$

- $\forall i, j \cdot \operatorname{rem}\left(\operatorname{clan}_{i}\right)=\operatorname{rem}\left(\operatorname{clan}_{j}\right)$,

- $\operatorname{shading}_{i} \in\{\square, \square\}$.

The word rem $\left(\right.$ overlap $\left._{i}\right)$ is called the line-order of $d$, denoted lo $(d)$; the set of letters in $l o(d)$ is called the lines of $d$, denoted $l(d)$; the word rem $\left(\right.$ clan $\left._{i}\right)$ is called the pointorder of d, denoted po $(d)$; and the set of letters in po $(d)$ is called the points of $d$, denoted $p(d)$. The point $p_{i}$ lies under overlap $p_{j}$, denoted $p_{i} \downarrow$ overlap $p_{j}$, whenever $p_{i}$ is unbarred in clan $n_{j}$. The set of overlaps in $d$ is denoted $\mathcal{O}_{d}$; and the set of all $w$ such that $\operatorname{rem}(w)=l o(d)$ is called the allowable overlaps of $d$, denoted $\mathcal{A O}_{d}$.

Much like repeated letters in overlaps, overlaps themselves can be repeated in a diagram. The drawn PaL diagram in Fig. 2 is, formally,

$d=[(A B,-, p q, \square),(A \bar{B},-, p \bar{q}, \square),(\bar{A} \bar{B}, \cdots \cdot \bar{p} \bar{q}, \square)]$.

In this diagram, $l(d)=\{A, B\}$ and $p(d)=\{p, q\}$. The following, however, is not a PaL diagram:

$$
[(A B C D,-, a b, \square),(A B \bar{C}, \cdots, \bar{a} \bar{b}, \square)]
$$

since $\operatorname{rem}(A B C D)=A B C D \neq A B C=\operatorname{rem}(A B \bar{C})$.

Definition 5 Given a unitary PaL diagram $d$, we call the set $\mathcal{O}_{d}(-)=\left\{O_{i} \in \mathcal{O}_{d}:\right.$ type $\left._{i}=-\right\}$ the solid overlaps of $d$; we call the set $\mathcal{O}_{d}(\square)=\left\{O_{j} \in \mathcal{O}_{d}:\right.$ sh $\left.h_{i}=\square\right\}$ the shaded overlaps of $d$; we call the set $\mathcal{P}_{d}(O)=\{p \in p(d)$ : $p \downarrow O\}$ the points lying under overlap $O$ in $d$; and we call the set $\mathcal{O}_{d}(p)=\left\{O \in \mathcal{O}_{d}: p \downarrow O\right\}$ the overlaps over point $p$.

We can then build up PaL diagrams using normal logical connectives and unitary $\mathrm{PaL}$ diagrams:

Definition 6 A PaL diagram is defined inductively as follows:

- if $d$ is a unitary PaL diagram then d is a PaL diagram;

- if $d_{1}$ is a PaL diagram then $\neg d_{1}$ is a PaL diagram where $l\left(\neg d_{1}\right)=l\left(d_{1}\right)$ and $p\left(\neg d_{1}\right)=p\left(d_{1}\right)$;

- if $d_{1}$ and $d_{2}$ are PaL diagrams and $\diamond \in\{\wedge, \vee, \Rightarrow\}$ then $\left(d_{1} \diamond d_{2}\right)$ is a PaL diagram where $l\left(d_{1} \diamond d_{2}\right)=$ $l\left(d_{1}\right) \cup l\left(d_{2}\right)$ and $p\left(d_{1} \diamond d_{2}\right)=p\left(d_{1}\right) \cup p\left(d_{2}\right)$;

Given a unitary PaL diagram, we now show how to draw that diagram. The process will produce a drawn diagram where all overlaps have equal length, although it is a simple matter to drop this restriction. 
Definition 7 Given a unitary PaL diagram

$$
d=\left[\left(O_{1}, \text { type }_{1}, c l_{1}, s h_{1}\right), \ldots,\left(O_{n}, \text { type }_{n}, c l_{n}, s h_{n}\right)\right]
$$

we draw the concrete diagram for $\boldsymbol{d}$ as follows:

1. Write the word $U \cdot l o(d) \cdot p o(d)$ vertically downwards, followed by a vertical line of equivalent length.

2. Draw

$\left(O_{1}\right.$, type $\left._{1}, c l_{1}, s h_{1}\right)=\left(L_{1} \ldots L_{m}\right.$, type $\left._{1}, p_{1} \ldots p_{k}, s h_{1}\right)$

as follows:

- If type t $_{1}=-$ then draw a solid horizontal line of length 1 unit against $U$ and, for each $j=$ $1, \ldots, m$, if $L_{j}$ is unbarred in $O_{1}$ draw a solid horizontal line against the letter $L_{j}$ of length 1 unit.

- If type t $_{1}=\ldots$ then draw a dashed horizontal line of length 1 unit against $U$ and, for each $j=$ $1, \ldots, m$, if $L_{j}$ is unbarred in $O_{1}$ draw a dashed horizontal line against the letter $L_{j}$ of length 1 unit.

- For $j=1, \ldots, k$, if $p_{j}$ is unbarred then draw • in the middle of the horizontal space of width 1 unit against the letter $p_{j}$.

- If $s h_{1}=$ then shade the entire vertical column one unit wide.

For each $i=2, \ldots, n$, repeat the process as for

$$
\left(O_{1}, \text { type }_{1}, \mathrm{cl}_{1}, \mathrm{sh}_{1}\right),
$$

moving along $i-1$ units before starting to draw lines (resp. $i-\frac{1}{2}$ units for points).

3. Draw a box around the constructed elements.

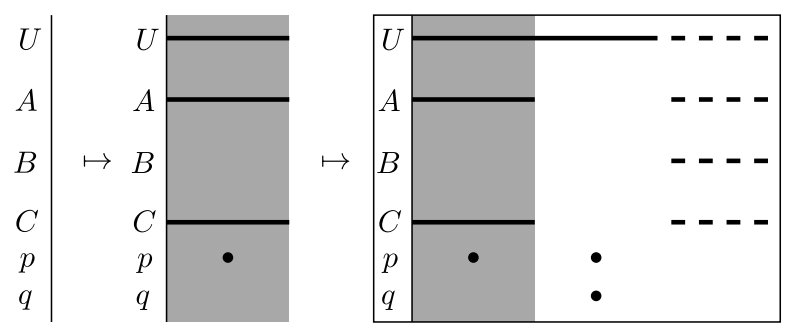

Figure 4. Drawing a PaL diagram

As an example, consider the unitary PaL diagram

$$
[(A \bar{B} C,-, p \bar{q}, \square),(\bar{A} \bar{B} \bar{C},-, p q, \square),(A B C, \cdots, \bar{p} \bar{q}, \square)] .
$$

The drawing stages for this diagram are shown in Fig. 4.
Given drawings of unitary PaL diagrams, we can combine them to form drawings of more general diagrams. Diagrammatically, negation is represented by crossing the diagram out, conjunction by juxtaposition, disjunction by drawing a horizontal line segment between diagrams (as in Shin's Venn-II system [12]) and implication by drawing a horizontal, single-headed arrow $(\Rightarrow)$ between diagrams. Concrete representations of these constructions are illustrated in Fig. 5, showing

$$
\neg[(A \bar{B} C,-, p \bar{q}, \square)]
$$

(c) $\quad[(\bar{A} \bar{B} \bar{C},-, \bar{p} q, \square)] \vee[(A B C,-, p q, \square)]$, and

(d) $\quad[(\bar{A} \bar{B} \bar{C},-, \bar{p} q, \square)] \Rightarrow[(\bar{A} B C,-, p \bar{q}, \square)]$.

We now have the syntax for PaL diagrams. We give them meaning in a natural way, given we are using them as representations of sets and elements. The lines and points are interpreted as subsets and elements of some universe, respectively. Formally:

Definition 8 An interpretation is a pair $(\mathcal{U}, I)$ where $\mathcal{U}$ is called the universal set and $I$ the function $I: \mathcal{L} \rightarrow \mathbb{P}(\mathcal{U})$ ensures $I(A) \subseteq \mathcal{U}$. The function I can be extended to interpret barred letters and overlaps as follows:

- for each letter, $A, I(\bar{A})=\mathcal{U}-I(A)$, and

- for each overlap $O$,

$$
I(O)=\bigcap_{A \in \mathcal{L}_{u}} I(A) \cap \bigcap_{A \in \mathcal{L}_{b}} I(\bar{A})
$$

where $\mathcal{L}_{u}$ is the set of letters which appear unbarred in $O$ and $\mathcal{L}_{b}$ is the set of letters which appear as barred letters in $O$.

If $\mathcal{U}$ is finite the size of the interpretation is $|\mathcal{U}|$.

Interpretations that agree with the intended meaning of a diagrams are called the diagram's models:

\section{Definition 9 Let}

$$
d=\left[\left(O_{1}, \text { type }_{1}, \operatorname{cl}_{1}, \text { sh }_{1}\right), \ldots,\left(O_{n}, \text { type }_{n}, \operatorname{cl}_{n}, s h_{n}\right)\right]
$$

be a unitary PaL diagram. An interpretation $\mathcal{I}=(\mathcal{U}, I)$ is a model for $d$, denoted $\mathcal{I}=d$, whenever there exists a function, namely $\Phi: \mathcal{P} \rightarrow \mathcal{U}$, mapping points to elements of $\mathcal{U}$ satisfying:

1. Point-location condition: each point maps to an element in the set represented by an overlap under which the point lies:

$$
\bigwedge_{p \in p(d)}\left(\Phi(p) \in \bigcup_{O_{i} \in \mathcal{O}_{d}(p)} I\left(O_{i}\right)\right) .
$$




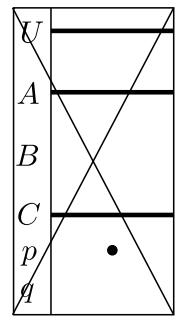

(a)

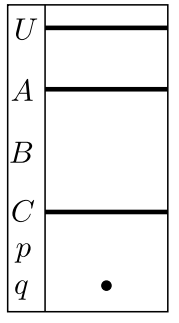

(b)

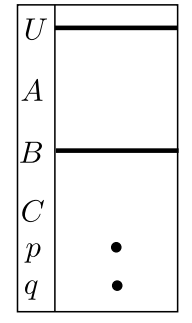

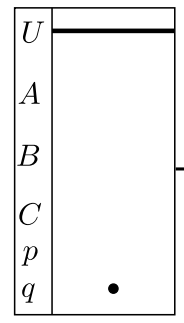

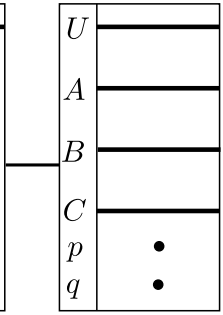

(c)
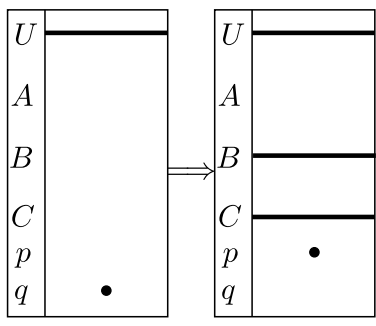

$(d)$

Figure 5. Components of PaL diagrams

2. Point-distinctness condition: no two distinct points map to the same element:

$$
\bigwedge_{p_{i}, p_{j} \in p(d)}\left(p_{i} \neq p_{j} \Rightarrow \Phi\left(p_{i}\right) \neq \Phi\left(p_{j}\right)\right) .
$$

3. Solid-overlap condition: Solid overlaps represent non-empty sets:

$$
\bigwedge_{O \in \mathcal{O}_{d}(-)}(I(O) \neq \emptyset)
$$

4. Overlap-absence condition: Overlaps that could be present given the line labels used represent empty sets:

$$
\bigwedge_{O \in \mathcal{A O}_{d}-\mathcal{O}_{d}}(I(O)=\emptyset)
$$

5. Shaded-overlap condition: in a shaded overlap, all elements are represented by points:

$$
\bigwedge_{O_{i} \in \mathcal{O}_{d}(\amalg)}\left(I\left(O_{i}\right) \subseteq\left\{\Phi\left(p_{i}\right): p_{i} \in c l_{i}\right\}\right) .
$$

\section{If $\mathcal{I}$ is a model for $d$ then $\mathcal{I}$ satisfies $d$.}

As an example, take the final diagram in figure 4 , and consider the interpretation $(\mathcal{U}, I)$ where $\mathcal{U}=$ $\{1,2,3\}, I(A)=\{1,3\}, I(B)=\{3\}, I(C)=$ $\{1,3\}, \Phi\left(p_{1}\right)=\{1\}, \Phi\left(p_{2}\right)=\{2\}$. We show that $(\mathcal{U}, I) \models$ $d$. For every solid overlap present in $d$, we have $I(O) \neq$ $\emptyset$. To illustrate, the second overlap is $\bar{A} \bar{B} \bar{C}$, giving interpretation $(\mathcal{U}-I(A)) \cap(\mathcal{U}-I(B)) \cap(\mathcal{U}-I(C))=$ $\{2\} \cap\{1,2\} \cap\{2\}=\{2\} \neq \emptyset$. Further, the overlap $\bar{A} B \bar{C}$ (amongst others) is absent. The interpretation of this overlap is $(\mathcal{U}-I(A)) \cap I(B) \cap(\mathcal{U}-I(C))=\{2\} \cap\{3\} \cap\{2\}=\emptyset$, as required. Consider the point $p_{2}$, lying under the overlap $\bar{A} \bar{B} \bar{C}$. We have already seen the interpretation of this overlap is $\{2\}$, and since $\Phi\left(p_{2}\right) \in\{2\}$, we have that the point-location condition is satisfied for $p_{2}$. Now, to satisfy the point-location condition for $p_{1}$, we require that $\Phi\left(p_{1}\right) \in$ $\{1,3\} \cup\{2\}$, which holds. The shaded-overlap condition for the first overlap requires that $I(A) \cap(\mathcal{U}-I(B)) \cap I(C) \subseteq$ $\left\{\Phi\left(p_{1}\right)\right\}$. Now, since $\Phi\left(p_{1}\right)=1$, the condition is satisfied. All conditions are thus true, and so $(\mathcal{U}, I) \models d$.

Note that $(\mathcal{U}, I)$ is not the only model for $d$. There is no maximum cardinality restriction on the number of elements in $I(\bar{A} \bar{B} \bar{C})$. Thus, keeping the function $I$ the same, but changing $\mathcal{U}$ to $\{1,2,3,4\}$ will still be a model for $d$, except now $I(\bar{A} \bar{B} \bar{C})=\{2,4\}$. By contrast, we cannot add extra elements to $I(A \bar{B} C)$, since otherwise we would violate the shaded overlap condition. This observation that the model sets for unshaded overlaps can be extended, but the sets for shaded overlaps cannot necessarily be extended, will be crucial in section 5 .

The interpretation $(\{1,2\}, I)$ where $I(A)=I(B)=$ $I(C)=\emptyset, \Phi(p)=\{1\}, \Phi(q)=\{2\}$ is likewise a model for $d$. This model illustrates the shaded-overlap condition requiring a subset relation, rather than equality. For, the interpretation of the first overlap is $\emptyset$, since $p$ is interpreted as lying under the second overlap, so clearly $I(\bar{A} B \bar{C})=$ $\emptyset \neq\{1\}$.

Consider the unitary diagram $d=[(A,-, \lambda, \square)]$, in other words the fully shaded diagram with one solid overlap, $A$, with no points lying under it. Consider further the an interpretation $\mathcal{I}$. The solid overlap presence condition tells us that $I(A) \neq \emptyset$. By contrast, the shaded overlap condition tells us $I(A)$ is subset of the interpretations of the points lying under the overlap. Since no points lie under the overlap $A$, we have that $I(A) \subseteq \emptyset$. Thus, the conditions cannot all be true, and so $\mathcal{I}$ is not a model for $d$, that is we say $d$ is unsatisfiable. There are many unsatisfiable diagrams, but the canonical unsatisfiable diagram, denoted $d_{\perp}$, is defined to be:

$$
d_{\perp}=[(\lambda,-, \lambda, \square)] .
$$

We need to define models for arbitrary $\mathrm{PaL}$ diagrams. This is straightforward:

Definition 10 Given an interpretation $\mathcal{I}=(\mathcal{U}, I)$ and a non-unitary PaL diagram $d$, we say that $\mathcal{I}$ is a model for $d$, 
denoted $\mathcal{I} \models d$, based on the structure of $d$ :

1. if $d \equiv \neg d_{1}$, then $\mathcal{I} \models d$ whenever $\mathcal{I} \not \models d_{1}$,

2. if $d \equiv d_{1} \vee d_{2}$, then $\mathcal{I} \models d$ whenever $\mathcal{I}=d_{1}$ or $\mathcal{I} \mid=d_{2}$,

3. if $d \equiv d_{1} \wedge d_{2}$, then $\mathcal{I} \models d$ whenever $\mathcal{I} \models d_{1}$ and $\mathcal{I}=d_{2}$, and

4. if $d \equiv d_{1} \Rightarrow d_{2}$, then $\mathcal{I} \mid=d$ whenever $\mathcal{I} \mid=d_{1}$ implies $\mathcal{I} \mid=d_{2}$.

Each dot against a point label represents disjunctive information. For example, in the right-most diagram of Fig. 5 , there are two dots for the point $p$. This arrangement means that the point $p$ will be interepreted either as the first dot, or the second, but not both. Conjunctive information about points, meanwhile, will be represented by duplicate point labels. Wherever a point-order for a diagram contains two instances of the same letter, say $p$, then unless there is some clan where both instances of $p$ are unbarred, then the diagram will necessarily represent a contradiction.

The use of multiple dots against a single point label to represent disjunction provides a compact notation. If points were singular (could only consist of a single dot), then disjunction would have to be represented as a disjunction of unitary PaL diagrams. Consider the simple case of a diagram for Venn-2, together with a pair of points $p$ and $q$ where the only information we know is that $p$ and $q$ are distinct points. In other words, we do not know in which sets their interpretations are. This situation is illustrated in Fig. 6. If points were instead singular, then we would need 10 unitary diagrams connected by disjunctions to represent the same information. Suppose that the interpretation of $p$ is an element of the intersections of the interpretations of $A$ and not $B$. Then, there are 4 possible locations for the interpretation of $q$, requiring 4 separate representative diagrams (the first four components of Fig. 7). Suppose, instead, that the interpretation of $p$ is an element of the intersection of the interpretations of $A$ and $B$. Recalling that the points represent variables, not constants, there are now only 3 possible locations for the interpretation of $q$. Continuing in this way, we see that there are $4+3+2+1=10$ different unitary diagrams needed when we restrict points to be singular. The diagram in Fig. 7 represents the same information as the in Fig. 6 yet the latter is more compact than the former. In general, where $m$ points each lie on $n$ overlaps, then the number of disjuncts needed if each point is to be singular is $O\left(n^{m}\right)$, although proving this simple result is outside the scope of this paper.

\section{Monadic First-Order Logic with Equality}

To show that $\mathrm{PaL}$ diagrams are exactly as expressive as monadic first-order logic with equality $(\operatorname{MFOL}[=])$, we aim

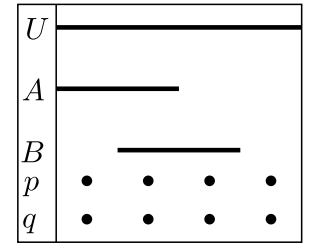

Figure 6. Points as disjunctive information

to provide translations between sentences in MFOL $[=]$ and diagrams. In order to do this, we first give a brief survey of some necessary results about MFOL $[=]$.

A monadic predicate symbol is one which takes a single argument. For example, $L(x)$ uses the monadic predicate symbol $L$, whereas $D(x, y)$ uses the dyadic predicate symbol $D$. MFOL $[=]$ has only one special dyadic predicate, that of equality. We use $\mathcal{L}$ as the set of monadic predicate symbols, thus treating the line labels as monadic predicates. Further, we take the set of variables to include all points in $\mathcal{P}=\left\{p_{1}, p_{2}, \ldots\right\}$, and sometimes use the more usual $x, y, \ldots$ as well. Using points as variables makes definitions later in the paper more straightforward (variables will arise from points in our translations). Using $x$ and $y$ as variables distinguishes them from those arising from points.

Briefly, formulae in $\operatorname{MFOL}[=]$ are defined using monadic predicate symbols, variables, $=$, logical connectives and quantifiers in the standard way. Given a formula, $\psi$, if $\psi$ has no free variables (i.e. variables that are not bound by a quantifier) then $\psi$ is a sentence. Every formula can be turned into a semantically equivalent sentence by binding the free variables with universal quantifiers. As we use the standard syntax and semantics of MFOL $[=]$ full details of the syntax and semantics are omitted; unfamiliar readers are referred to [2]. Firstly, we define the notion of expressive equivalence:

Definition 11 A diagram and a sentence are expressively equivalent whenever they have the same set of models.

In order to prove the expressive equivalence between PaL diagrams and MFOL $[=]$, we compare the model sets of each. As an example, consider the MFOL $[=]$ sentence $S=\exists x . A(x)$. Take the interpretation $\mathcal{I}=$ $(\{1,2\},\{(A,\{1\}), \ldots\})$. This interpretation is a model for $S$, whereas any interpretation with $I(A)=\emptyset$ is not: $S$ stipulates that any interpretation of $A$ must be non-empty.

We briefly summarise some results about models for $\operatorname{MFOL}[=]$ contained in [16]. In particular, we include the definitions that are needed to state a key theorem about $\operatorname{MFOL}[=]$ sentences, encapsulating the fact that each sentence, $S$, has a finite set of 'small' models (formally defined later) that can be used to generate all models of $S$. These small models are crucial for constructing a diagram with 

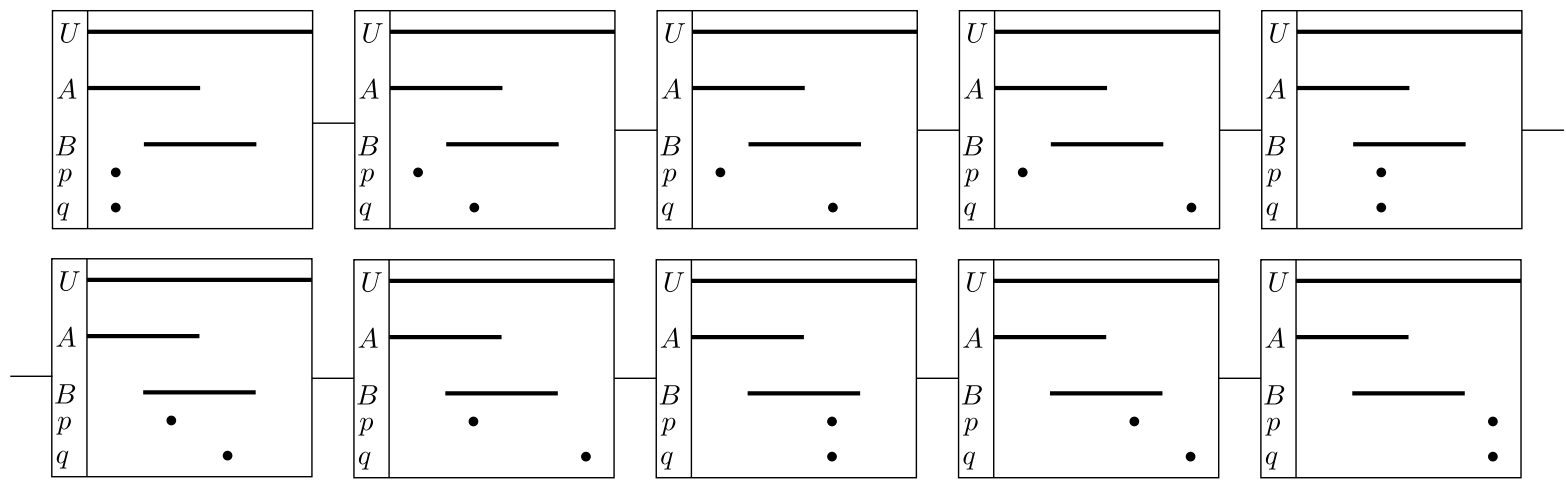

Figure 7. Disjunctive blow-up

the same meaning as $S$. Like diagrams, each sentence $S$ in MFOL $[=]$ may have many models. The first step is to identify the interpretation of sets of predicate symbols, akin to the interpretation of overlaps:

Definition 12 Let $\mathcal{I}$ be a interpretation with universal set $\mathcal{U}$ and let $X$ and $Y$ be finite subsets of $\mathcal{L}$. The predicate intersection set in $\mathcal{I}$ with respect to $X$ and $Y$, denoted $P I(\mathcal{I}, X, Y)$, is given by:

$$
P I(\mathcal{I}, X, Y)=\bigcap_{L_{i} \in X} I\left(L_{i}\right) \cap \bigcap_{L_{i} \in Y}\left(\mathcal{U}-I\left(L_{i}\right)\right)
$$

where $\bigcap_{L_{i} \in \emptyset} I\left(L_{i}\right)=\bigcap_{L_{i} \in \emptyset}\left(\mathcal{U}-I\left(L_{i}\right)\right)=\mathcal{U}[16]$.

Given a sentence $S$, denote by $q(S)$ and $P(S)$ the quantifier rank of $S$ [5] and set of predicates in $S$, respectively; recall, the quantifier rank of $S$ is the maximum number of nested quantifiers in $S$. A sentence with quantifier rank of $n$ can contain at most $n$ distinct variables within the body of the sentence. Now, to limit the cardinality of a predicate intersection set to, say, $m$ we need $m+1$ distinct variable names. To see this, consider the sentence $\exists p_{1} \cdot \forall p_{2} \cdot p_{1}=p_{2}$. Any model for this sentence must have size 1 , since the sentence tells us some element exists, and every element is equal to it. This argument is easily extended to arbitrary $m>1$. Given a model for $S$ and a predicate intersection, $P I$, set with cardinality at least $q(S)$, elements can be added to $P I$ and the resulting interpretation is still a model for $S$. By contrast, if $P I$ has cardinality less than $q(S)$ then elements cannot necessarily be added to it. Given any model for $S$, we can identify which predicate intersection sets can safely be extended with extra elements. Formally:

Definition 13 Let $S$ be a sentence and let $\mathcal{I}_{1}$ be a model for $S$. An $\boldsymbol{S}$-extension of $\mathcal{I}_{1}$ is an interpretation, $\mathcal{I}_{2}$, such that for each subset $X$ of $P(S)$ :

$$
P I\left(\mathcal{I}_{1}, X, P(S)-X\right) \subseteq P I\left(\mathcal{I}_{2}, X, P(S)-X\right)
$$

with equality whenever $\left|P I\left(\mathcal{I}_{1}, X, P(S)-X\right)\right|<q(S)$ [16].

Definition 14 Let $S$ be a sentence and $\mathcal{I}$ be a model for $S$. If the cardinality of $\mathcal{I}$ is at most $2^{|P(S)|} q(S)$ then we say $\mathcal{I}$ is a small model for $S$ [16].

Given a sentence $S$ we have that $q(S)$ and $2^{|P(S)|}$ are finite, and so there are finitely many candidate interpretations which can be small models for $S$. We say two interpretations $\mathcal{I}_{1}=\left(\mathcal{U}_{1}, I_{1}\right)$ and $\mathcal{I}_{2}=\left(\mathcal{U}_{2}, I_{2}\right)$ are isomorphic restricted to $P(S)$ iff there exists an isomorphism between $\mathcal{I}_{1}$ and $\mathcal{I}_{2}$ when the domains of $I_{1}$ and $I_{2}$ are both restricted to $P(S)$.

Definition 15 Let $S$ be a sentence. A set of small models, $c(S)$, is called a classifying set of models for $S$ if for each small model $m_{1}$ for $S$, there exists a unique $m_{2} \in c(S)$ such that $m_{1}$ and $m_{2}$ are isomorphic restricted to $P(S)$ [16].

In other words, a classifying set for $S$ is the smallest possible set of small models for $S$. We can create $S$-extensions of the small models for $S$ to create more models for $S$. Such extensions form a set called the cone:

Definition 16 The cone of $\mathcal{I}_{1}$ given $S$, denoted cone $\left(\mathcal{I}_{1}, S\right)$, is a class of interpretations such that $\mathcal{I}_{2} \in \operatorname{cone}\left(\mathcal{I}_{1}, S\right)$ iff $\mathcal{I}_{2}$ is isomorphic to some $S$-extension of $\mathcal{I}_{1}[16]$.

Finally, we have the key theorem needed for our expressiveness result:

Theorem 1 Let $S$ be a sentence and let $c(S)$ be a classifying set of models for $S$. Then $\bigcup_{m \in c(S)} \operatorname{cone}(m, S)$ is precisely the set of models for $S$ [16]. 


\section{Sentences for diagrams}

To show that every diagram can be turned into a sentence in $\operatorname{MFOL}[=]$, we translate the conditions from definition 9 into formulae in MFOL $[=]$. Given a unitary PaL diagram we need to know how to translate the overlaps, the clans and the shading into MFOL $[=]$ formulae. We first define a formula for an overlap, regardless of whether it is solid, dashed or shaded.

Definition 17 Let $O$ be an overlap with unbarred letters $\mathcal{L}_{u}$ and barred letters $\mathcal{L}_{b}$. The overlap formula for $O$, denoted $\mathcal{F}(O, x)$, is given by:

$$
\mathcal{F}(O, x)=\bigwedge_{L \in \mathcal{L}_{u}} L(x) \wedge \bigwedge_{L \in \mathcal{L}_{b}} \neg L(x) .
$$

We can now define the translation of a unitary PaL diagram to a $\mathrm{MFOL}[=]$ sentence.

\section{Definition 18 Let}

$$
d=\left[\left(O_{1}, \text { type }_{1}, c l_{1}, s h_{1}\right), \ldots,\left(O_{n}, \text { type }_{n}, c l_{n}, s h_{n}\right)\right]
$$

be a unitary PaL diagram, where $p(d)=\left\{p_{1}, \ldots, p_{s}\right\}$.

- The point-location formula for d, denoted $\mathcal{F}_{P L}(d)$, is a conjunction, over all points, of the disjunctive information given by each point of $d$ :

$$
\mathcal{F}_{P L}(d)=\bigwedge_{p_{i} \in p(d)}\left(\bigvee_{O \in \mathcal{O}_{d}\left(p_{i}\right)} \mathcal{F}\left(O, p_{i}\right)\right) .
$$

- The point-distinctness formula for $d$, denoted $\mathcal{F}_{P D}(d)$, is a conjunction, over all distinct points, of inequalities:

$$
\mathcal{F}_{P D}(d)=\bigwedge_{p_{i}, p_{j} \in p(d) \wedge i \neq j} p_{i} \neq p_{j}
$$

- The solid-overlap formula for d, denoted $\mathcal{F}_{S o O}(d)$, is a conjunction, over all solid overlaps, of the existential formulae:

$$
\mathcal{F}_{S o O}(d)=\bigwedge_{O \in \mathcal{O}_{d}(-)} \exists x \cdot \mathcal{F}(O, x) .
$$

- The overlap-absence formula for $d$, denoted $\mathcal{F}_{O A}(d)$, is a conjunction, over all allowable overlaps that are absent from $d$, of negated existential formulae stating that no elements lie under the absent overlaps:

$$
\mathcal{F}_{O A}(d)=\bigwedge_{O \in \mathcal{A O}_{d}-\mathcal{O}_{d}} \neg \exists x . \mathcal{F}(O, x)
$$

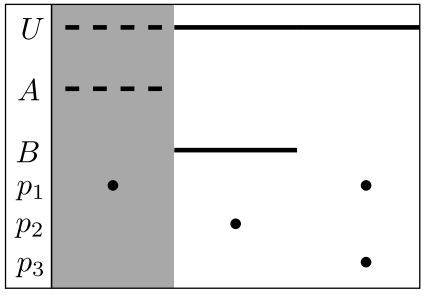

Figure 8. A PaL diagram to be translated to MFOL $[=]$

- The shaded-overlap formula for d, denoted $\mathcal{F}_{S h O}(d)$, is a conjunction, over all shaded overlaps, of universal formulae stating that if an element lies under an overlap, then it must be one of the points lying under that overlap:

$$
\mathcal{F}_{S h O}(d)=\bigwedge_{O \in \mathcal{O}_{d}(\varpi)}\left(\forall y \cdot\left(\mathcal{F}(O, y) \Rightarrow\left(\bigvee_{p \in \mathcal{P}_{d}(O)} y=p\right)\right)\right)
$$

The MFOL $[=]$ sentence for $d$, denoted $\mathcal{S}(d)$, is then given by:

$$
\mathcal{S}(d)=\exists p_{1}, \ldots, p_{s} . \mathcal{F}_{P L}(d) \wedge \mathcal{F}_{P D}(d) \wedge \mathcal{F}_{S o O}(d) \wedge \mathcal{F}_{S h O}(d) .
$$

Note that, if type ${ }_{i}=\cdots$ for some overlap then the overlap itself gives us no information (although the points lying under it might). Hence, there is no formula created from the dashed overlaps, just as no condition arose in definition 9.

Consider the diagram $d$ in figure 8 . We create an MFOL $[=]$ sentence for this diagram using the following formulae:

$$
\begin{aligned}
& \mathcal{F}_{P L}(d)=\left(A\left(p_{1}\right) \wedge \neg B\left(p_{1}\right)\right) \vee\left(\neg A\left(p_{1}\right) \wedge \neg B\left(p_{1}\right)\right) \wedge \\
&\left(\neg A\left(p_{2}\right) \wedge B\left(p_{2}\right)\right) \wedge \\
&\left(\neg A\left(p_{3}\right) \wedge \neg B\left(p_{3}\right)\right), \\
& \mathcal{F}_{P D}(d)=\left(p_{1} \neq p_{2} \wedge p_{1} \neq p_{3} \wedge p_{2} \neq p_{3}\right), \\
& \mathcal{F}_{S o O}(d)= \exists x .(\neg A(x) \wedge B(x)) \wedge \exists x .(\neg A(x) \wedge \neg B(x)), \\
& \mathcal{F}_{O A}(d)=\neg \exists x .(A(x) \wedge B(x)), \\
& \mathcal{F}_{S h O}(d)=\forall y .\left((A(y) \wedge \neg B(y)) \Rightarrow\left(y=p_{1}\right)\right) .
\end{aligned}
$$

Thus the sentence for $d$ is:

$S(d)=\exists p_{1} p_{2} p_{3} . \mathcal{F}_{P L}(d) \wedge \mathcal{F}_{P D}(d) \wedge \mathcal{F}_{S o O}(d) \wedge \mathcal{F}_{O A}(d) \wedge \mathcal{F}_{S h O}(d)$.

Theorem 2 Every unitary PaL diagram $d$ is expressively equivalent to $S(d)$.

The proof is straightforward: each part of the sentence corresponds to an encoding in $\mathrm{MFOL}[=]$ of the conditions in definition 9. It is also immediate how to extend the result to arbitrary $\mathrm{PaL}$ diagrams: 
Definition 19 Let d be a PaL diagram. The MFOL $[=]$ sentence for $\boldsymbol{d}$, denoted $S e n(d)$, is given by induction on the structure of $d$ :

- if $d$ is a unitary diagram then $\operatorname{Sen}(d)$ is already defined,

- if $d$ is $\neg d_{1}$, then $\operatorname{Sen}(d)=\neg \operatorname{Sen}\left(d_{1}\right)$,

- if $d$ is $d_{1} \diamond d_{2}$, where $\diamond \in\{\wedge, \vee, \Rightarrow\}$, then $\operatorname{Sen}(d)=$ $\left(\operatorname{Sen}\left(d_{1}\right) \diamond \operatorname{Sen}\left(d_{2}\right)\right)$.

Theorem 3 Every PaL diagram d is expressively equivalent to $\operatorname{Sen}(d)$.

\section{Diagrams for sentences}

In order to construct a diagram for the sentence $S$, we need only construct diagrams for the small models in $c(S)$, and take the disjunction of these diagrams. In what follows we let $P I_{X, \mathcal{I}, S}=|P I(\mathcal{I}, X, P(S)-X)|$. The process for drawing a diagram for each small model is straightforward. First, if $c(S)$ contains the interpretation with $|\mathcal{I}|=0$, then this model gives rise to the empty diagram $d_{\emptyset}=[(\lambda, \cdots, \lambda, \square)]$. This diagram contains no points or lines other than the $U$ line, and the shading asserts that the universe is empty. For non-empty models, we assign solid overlaps to non-empty predicate intersection sets and we create the same number of points lying under this overlap as the cardinality of the predicate intersection set. We shade those overlaps where the associated predicate intersection set has smaller size than the quantifier rank of the sentence. Formally:

Definition 20 Let $\mathcal{I}$ be a small model for a $M F O L[=]$ sentence $S$ and suppose $|\mathcal{I}|=m$ and $|P(S)|=n$ where $P(S)=\left\{L_{1}, \ldots, L_{n}\right\}$. Let the set $\{X: X \subseteq P(S) \wedge$ $\left.P I_{X, \mathcal{I}, S}>0\right\}=\left\{X_{1}, \ldots, X_{N}\right\}$ be ordered. The PaL diagram d representing $\mathcal{I}$ given $S$, denoted $\mathcal{D}(\mathcal{I}, S)=d$ is defined as follows:

1. If $|\mathcal{I}|=0$, then $d=d_{\emptyset}$.

2. Otherwise, the line labels are the predicate symbols in $P(S)$ and set $l o(d)=L_{1} \ldots L_{n}$.

3. There is one point label for each element of $\mathcal{U}$ :

$$
p(d)=\left\{p_{1}, \ldots, p_{m}\right\}
$$

and set $p o(d)=p_{1} \ldots p_{m}$.

4. For $i=1, \ldots, N$ construct the overlap $\left(L_{1} \ldots L_{n},-, p_{1} \ldots p_{m}, s h_{i}\right)$ where:

- $L_{j}$ is unbarred iff $L_{j} \in X_{i}$,
- $p_{k}$ is unbarred iff:

$$
\sum_{j=1}^{i-1} P I_{X_{j}, \mathcal{I}, S}<k \leq \sum_{j=1}^{i} P I_{X_{j}, \mathcal{I}, S}
$$

where we define $\sum_{j=1}^{0} P I_{X_{j}, \mathcal{I}, S}=0$,

- $s h_{i}=\square$ iff $P I_{X_{i}, \mathcal{I}, S}<q(S)$.

We illustrate the process of determining whether points are unbarred with an example. Suppose a model has $X_{1}, X_{2}$ and $X_{3}$ as the only sets where $P I_{X, \mathcal{I}, S}>0$, and $P I_{X_{1}, \mathcal{I}, S}=3, P I_{X_{2}, \mathcal{I}, S}=4$ and $P I_{X_{3}, \mathcal{I}, S}=2$. The values $k$ can take such that $P I_{X_{1}, \mathcal{I}, S}<k \leq P I_{X_{1}, \mathcal{I}, S}+$ $P I_{X_{2}, \mathcal{I}, S}$ are $4,5,6$ and 7 . Then the unbarred points in the second clan would be $p_{4}, p_{5}, p_{6}$ and $p_{7}$, meaning $p_{1}, p_{2}, p_{3}, p_{8}$ and $p_{9}$ would be barred. A consequence of definition 20 is that every point lies on exactly one overlap.

Having defined the diagrams representing an interpretation given a sentence, we now define the diagrams for the sentence:

Definition 21 Given an $M F O L[=]$ sentence $S$ with classifying models $c(S)$, the diagram representing $S$, denoted $\mathcal{D}(S)$, is given by:

$$
\mathcal{D}(S)=d_{\perp} \vee \bigvee_{\mathcal{I} \in c(S)} \mathcal{D}(\mathcal{I}, S)
$$

We demonstrate definition 21 using an example. One of the small models of the sentence $S=\exists x . A(x) \vee \forall x . A(x)$ is given by $\mathcal{I}=(\{1,2\}, I)$ where $I(A)=\{1\}$. There is a single predicate symbol in $S$, and so the line order for $d$ is simply as $A$. Furthermore, the set $X_{1}=\{A\}$ and $X_{2}=\emptyset$ are the only sets for which $P I_{X, \mathcal{I}, S}>0$. So, the number of overlaps in $\mathcal{D}(\mathcal{I}, S)$ is 2 . In this example, $|\mathcal{I}|=2$ so we require 2 points in each clan. The point order of $d$ is given by $p_{1} p_{2}$.

The first overlap, $\left(A,-, p_{1} \bar{p}_{2}, \square\right)$, is unshaded since $P I_{X_{1}, \mathcal{I}, S}=1 \geq q(S)=1$. Also, since $0<1 \leq 1, p_{1}$ is unbarred but $p_{2}$ is not. Similarly, the second overlap is $\left(\bar{A},-, \bar{p}_{1} p_{2}, \square\right)$.

The rest of the small models for $S$ are:

1. $\mathcal{I}_{1}=(\emptyset, \emptyset)$,

2. $\mathcal{I}_{2}=(\{1\},\{(A,\{1\})\})$,

3. $\mathcal{I}=\mathcal{I}_{3}=(\{1,2\},\{(A,\{1\})\})$, and

4. $\mathcal{I}_{4}=(\{1,2\},\{(A,\{1,2\})\})$.

The diagram for this sentence is given in figure 9 .

The first model, $\mathcal{I}_{1}$, comes from the vacuous satisfaction of $\forall x . A(x)$, giving rise to the empty diagram. We have already shown the diagram for the model $\mathcal{I}_{3}$, and the 


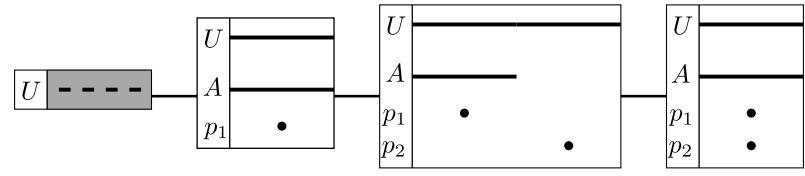

Figure 9. The PaL diagram for $\exists x . A(x) \vee$ $\forall x . A(x)$

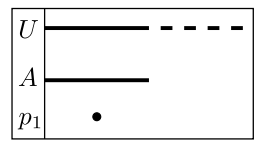

Figure 10. A more natural diagram for $\exists x . A(x)$

rest are left for the reader to verify. We note that the diagram in figure 9 could be considered a relatively natural $\mathrm{PaL}$ diagram for the sentence. We could write $\forall x . A(x)$ using the law of excluded middle: either no $x$ exists, or every $x$ has $A(x)$. Thus, the first two disjuncts of figure 9 , i.e. $d_{\emptyset} \vee\left[\left(A,-, p_{1}, \square\right)\right]$, represent the universal part of the sentence. The former covers the case where no $x$ exists (and so $\forall x . A(x)$ is trivially satisfied), and the latter covers the case where $\forall x . A(x)$ holds for non-empty universes. The existential part of the sentence can be thought of as follows: we know an $x$ exists such that $A(x)$, but this does not preclude other $y$ existing such that $\neg A(y)$. In order to capture this situation, we would use the diagram in figure 10 . The overlap $\left(\bar{A}, \cdots, \bar{p}_{1}, \square\right)$ tells us only that it is possible for such $y$ to exist, but does not necessitate its existence. We could thus replace the third and fourth components of figure 9 with the PaL diagram from figure 10.

The purpose of definitions 20 and 21 was not to produce the most natural diagrams for sentences (an interesting challenge for future work), however, but rather to demonstrate that every sentence could be translated into some PaL diagram.

Theorem 4 Let $S$ be a sentence. Then $\mathcal{D}(S)$ is expressively equivalent to $S$.

Proof. (Sketch) We show that definition 20 provides a diagram with models for $S$, and the corresponding proof for definition 21 follows immediately. Let $\mathcal{I}$ be a small model for $S$ in $c(S)$, and let $\mathcal{D}(\mathcal{I}, S)$ be constructed according to definition 20 . We show that any model $\mathcal{I}_{1} \in \operatorname{cone}(\mathcal{I}, S)$ is a model for $\mathcal{D}(\mathcal{I}, S)$, omitting the details of the converse.

If $\mathcal{I}_{1} \in \operatorname{cone}(\mathcal{I}, S)$ then it has been extended from $\mathcal{I}$ in such a way that

$$
P I(\mathcal{I}, X, P(S)-X)=P I\left(\mathcal{I}_{1}, X, P(S)-X\right)
$$

whenever $P I_{X, \mathcal{I}, S}<q(S)$, and

$$
P I(\mathcal{I}, X, P(S)-X) \subseteq P I\left(\mathcal{I}_{1}, X, P(S)-X\right)
$$

otherwise. Let $O$ be the overlap associated with the set $X$, and thus $|I(O)|=P I_{X, \mathcal{I}_{1}, S} \geq P I_{X, \mathcal{I}, S}$. There are $P I_{X, \mathcal{I}, S}$ distinct points lying under $O$ and at least $P I_{X, \mathcal{I}, S}$ distinct elements in $I(O)$, so we can thus assign each point lying under $O$ to a distinct element of $I(O)$ using a function $\Phi$. In this way, we have satisfied the point location and point distinctness conditions.

If $P I_{X, \mathcal{I}, S}<q(S)$ then $O$ is shaded and $|I(O)|=$ $P I_{X, \mathcal{I}, S}$. Since every point of $\mathcal{D}(\mathcal{I}, S)$ lies under exactly one overlap, and there are $P I_{X, \mathcal{I}, S}$ of them by the point distinctness and location conditions being satisfied we have that

$$
\bigcup_{p \in \mathcal{P}_{d}(O)} \Phi(p)=I(O)
$$

Trivially, then, $I(O) \subseteq \bigcup_{p \in \mathcal{P}_{d}(O)} \Phi(p)$, and thus the shaded overlap condition is satisfied.

If $P I_{Y, \mathcal{I}_{1}, S}=0$ then we draw no overlap, meaning $O \in \mathcal{A O}_{\mathcal{D}(\mathcal{I}, S)}-\mathcal{O}_{\mathcal{D}(\mathcal{I}, S) \text {. However, since we associate }}$ $O$ with $Y$, we also have $I(O)=\emptyset$, and thus the overlap absence condition is satisfied. Put another way, since we only draw an overlap when $P I_{X, \mathcal{I}_{1}, S} \neq 0$, every $X$ such that $P I_{X, \mathcal{I}_{1}, S} \neq 0$ is assigned to an overlap $O$ which has $I(O) \neq \emptyset$, satisfying the solid overlap presence condition. Then, the model $\mathcal{I}_{1}$ is also a model for $\mathcal{D}(\mathcal{I}, S)$, as required. Hence, by theorem 1 , every model for $S$ is a model for $\mathcal{D}(\mathcal{I}, S)$.

We have seen how any MFOL[=] sentence $S$ can be translated into a PaL diagram, and any $\mathrm{PaL}$ diagram can be translated into an MFOL $[=]$ sentence. Therefore:

Theorem 5 PaL diagrams and $M F O L[=]$ are equally expressive.

The main result of [16] was that spider diagrams are equally expressive as MFOL $[=]$. We thus have the following:

Theorem 6 PaL diagrams, MFOL $[=]$ and spider diagrams are all expressively equivalent.

\section{Conclusion and Further Work}

The results from $[3,11]$ demonstrated that linear diagrams have the potential to be an effective visual language in the areas where Euler and Venn diagrams are currently used. To explore and exploit this observation, we have extended linear diagrams to PaL diagrams by adding points. Moreover, we have formalised the syntax and semantics of $\mathrm{PaL}$ diagrams and shown they are capable of expressing 
exactly the same statements as MFOL $[=]$. As a corollary, we immediately have that PaL diagrams are exactly as expressive as spider diagrams which extend Euler diagrams. PaL diagrams provide a solid foundation for further development of linear-based notations, both in terms of expressiveness and reasoning.

There are a number of clear directions for further work. The creation of a system of inference rules for PaL diagrams is of particular interest. The reasoning rules for Euler-based diagrams with an equivalent level of expressiveness demonstrate that a sound and complete system of rules is possible. Given a sound and complete reasoning system for PaL diagrams, it should be possible to integrate these diagrams into a heterogeneous system such as Diabelli [17].

In the usability direction, we will seek layout guidelines for PaL diagrams that aid understanding. For example, to what extent is the ordering of the lines important? Is interleaving of the points and lines ever beneficial? By answering these questions, and others, empirically we seek to develop conditions akin to the well-formed conditions of Euler diagrams [15]. However, rather than these conditions being purely theoretical, to be tested empirically later, the empirical method will drive their development. In other words, we will develop a set of ease-of-understanding guidelines.

In terms of comparative usability, now that we have a system equivalent to spider diagrams, it is possible to extend the results of [3], which established linear diagrams' superiority over Euler diagrams, to notations equivalent to MFOL $[=]$. We hypothesise that PaL diagrams, when compared to spider diagrams, will retain the efficacy that linear diagrams have over Euler diagrams.

\section{References}

[1] F. Bellucci, A. Moktefi, and A.-V. Pietarinen. Diagrammatic autarchy. volume 1132. CEUR, 2014.

[2] S. Burris. Logic for Mathematics and Computer Science. Prentice Hall, 1998.

[3] P. Chapman, G. Stapleton, P. Rodgers, L. Micallef, and A. Blake. Visualizing Sets: An Emprical Comparison of Diagram Types, 2014.

[4] L. Couturat. Opuscules et fragments inédits de Leibniz. Felix Alcan, 1903.

[5] H.-D. Ebbinghaus and J. Flum. Finite Model Theory. Springer-Verlag, 1991.

[6] G. Englebretsen et al. Linear diagrams for syllogisms (with relationals). Notre Dame Journal of Formal Logic, 33(1):37-69, 1991.

[7] J. Gil, J. Howse, and S. Kent. Constraint diagrams: A step beyond UML. In Proceedings of TOOLS USA 1999, Santa Barbara, California, USA, pages 453-463. IEEE Computer Science Press, August 1999.

[8] H. Hofmann, A. Siebes, and A. Wilhelm. Visualizing Association Rules with Interactive Mosaic Plots. In Proceedings of the sixth ACM SIGKDD international conference on Knowledge discovery and data mining, pages 227-235. ACM, 2000.

[9] O. Lemon and I. Pratt. On the insufficiency of linear diagrams for syllogisms. Notre Dame Journal of Formal Logic, 39(4):573-580, 1998.

[10] P. Rodgers. A survey of Euler diagrams. J. Vis. Lang. Comput., 25(3):134-155, 2014

[11] Y. Sato and K. Mineshima. The Efficacy of Diagrams in Syllogistic Reasoning: A Case of Linear Diagrams. In $\mathrm{Di}$ agrammatic Representation and Inference, Lecture Notes in Computer Science, pages 352-355. Springer, 2012.

[12] S.-J. Shin. The Logical Status of Diagrams. Cambridge University Press, 1994.

[13] G. Stapleton and A. Delaney. Evaluating and generalizing constraint diagrams. Journal of Visual Languages and Computing, 19(4):499-521, 2008.

[14] G. Stapleton and J. Masthoff. Incorporating negation into visual logics: A case study using Euler diagrams. In Visual Languages and Computing 2007, pages 187-194. Knowledge Systems Institute, 2007.

[15] G. Stapleton, P. Rodgers, J. Howse, and J. Taylor. Properties of Euler diagrams. In Proceedings of Layout of Software Engineering Diagrams, pages 2-16. EASST, 2007.

[16] G. Stapleton, S. Thompson, J. Howse, and J. Taylor. The expressiveness of spider diagrams. Journal of Logic and Computation, 14(6):857-880, December 2004.

[17] M. Urbas and M. Jamnik. Heterogeneous Proofs: Spider Diagrams Meet Higher-Order Provers. In 2nd International Conference on Interactive Theorem Proving, pages 376$382,2011$.

[18] K. Wittenburg, T. Lanning, M. Heinrichs, and M. Stanton. Parallel Bargrams for Consumer-based Information Exploration and Choice. In Proceedings of the 14th annual ACM symposium on User interface software and technology, pages 51-60. ACM, 2001. 